\title{
MEDIA, SONDAGGI E SPESE ELETTORALI: LA NUOVA DISCIPLINA
}

\author{
di Carlo Fusaro
}

La legislazione elettorale di contorno: prospettive comparate

La legge 10 dicembre 1993 , n. 515 costituisce un vero e proprio «sistema elettorale di contorno». Pur constando di soli 20 articoli, si tratta di un testo di notevole complessità e assai eterogeneo. Questo saggio si propone di illustrarne genesi e contenuti, di mettere in luce alcune delle problematiche che ha sollevato e di esprimere qualche valutazione sulla sua prima applicazione in occasione delle elezioni del 1994.

L'importanza della c.d. legislazione elettorale di contorno (di contorno sia rispetto alla formula sia rispetto al sistema elettorale), con particolare riferimento alla disciplina di tutto ciò che concerne la fase che precede il momento in cui gli elettori sono chiamati alle urne per un voto (c.d. fase preparatoria), è stata colta da tempo sia in sede scientifica sia in sede legislativa. Ciò è confermato, in vari ordinamenti, da precise norme di diritto positivo'. Del resto, se «eleggere» non deve voler dire «scegliere a caso» ma «scegliere selezionando» (Sartori 1969, 89), ciò richiede che la struttura del processo di selezione non solo si articoli fra più soggetti (come prescrive comunque il principio pluralista), ma sia effettivamente competitiva: il che presuppone a sua volta che vi sia una tendenziale parità fra $i$ candidati e le forze politiche, funzionale a che ciascun elettore

Nello stendere questo articolo bo potuto avvalermi della eccellente analisi della legge 515/93, condotta sotto la guida del prof. Stefano Grassi, nel quadro del Seminario Tosi 1994, da Sebastiano Faro, Ginevra Cerrina Feroni, Roberta Ferrante, Lucia Russo e Biancamaria Sabatini, la cui sintesi sarà pubblicata nel prossimo «Quaderno» dell'Associazione per gli studi e le ricerche parlamentari: di ciò molto li ringrazio.

1 Il riferimento d'obbligo è al Regno Unito dove la prima normativa in materia di controllo delle spese elettorali e disciplina delle campagne elettorali risale ad oltre un secolo fa: correva l'anno 1883 (v. Troccoli 1991, 318; Gardini 1994, 57).

RIVISTA ITALIANA DI SCIENZA POLITICA / a. XXIV, n. 3, dicembre 1994 
possa essere informato e informarsi nel modo più completo sulle diverse opzioni a sua disposizione e, così, decidere liberamente. Ma vale anche una funzionalità inversa: poiché se la parità dei candidati è funzionale alla libertà di scelta dell'elettore, questa è a sua volta funzionale alla effettiva competitività del processo di selezione. L'una e l'altra costituiscono estrinsecazioni del principio democratico e sono condizioni per una scelta non casuale, ma, appunto, selettiva.

Ciò spiega perché assume rilievo, oltre al procedimento che culmina nell'atto con il quale la scheda votata entra nell'urna e nel successivo scrutinio, anche tutto ciò che viene prima nella misura in cui concorre al formarsi della volontà dell'elettore.

Se non che, quando si tratta di calare i principi astratti in scelte legislative concrete, le questioni si complicano.

Prima di tutto, un obiettivo può anche non conciliarsi con l'altro: tutto dipende dalle modalità concrete scelte per perseguirlo. A seconda di come la s'intende e di come si cerca di promuoverla, la parità dei soggetti in competizione può agevolmente andare a scapito della libertà di scelta dell'elettore, ove si pensi, per esempio, di garantirla attraverso disposizioni che riducano la qualità e la quantità della c.d. propaganda elettorale. Ciò vale anche per gli strumenti approntati a tutela della libertà di scelta dell'elettore, i quali possono nei fatti portare ad avvantaggiare alcuni dei soggetti in competizione a danno di altri e possono paradossalmente condurre a limitare le risorse informative sulla base delle quali si forma la sua volontà.

In secondo luogo, spesso il legislatore ha affidato alla disciplina della fase precedente il voto ulteriori obiettivi: ad esempio la tutela della pubblica tranquillità (e dell'ordine pubblico) in campagna elettorale; ovvero il contenimento delle spese elettorali, volto, oltre che a favorire condizioni di parità, anche ad evitare che $i$ soggetti in competizione siano prima o poi indotti in pericolose tentazioni.

$\mathrm{Ma}$, soprattutto, la disciplina delle campagne elettorali, proprio in ragione delle soluzioni individuate dal legislatore per perseguire gli obiettivi di cui si diceva, comporta una serie di disposizioni che incidono inevitabilmente sulla condizione giuridica di una pluralità di soggetti limitandoli, in più d'un caso, nell'esercizio di diritti a loro volta costituzionalmente tutelati: si pensi alle norme sull'accesso e la pubblicità sui media, per esempio. Esse incidono, in forme giuridicamente differenziate, sulla posizione soggettiva (a) dei soggetti titolari di imprese edi- 
toriali (stampa, radio, televisione e quant'altro) e di chi opera all'interno di esse (a partire dai giornalisti); (b) dei soggetti protagonisti della competizione politica (candidati, partiti, loro sostenitori); ( $c$ ) di tutti i cittadini. E lo fanno toccando situazioni giuridiche che in ogni ordinamento a derivazione liberaldemocratica sono connesse a valori di primaria rilevanza costituzionale: dalla libertà di manifestazione del pensiero alla libertà di voto (concepita in senso lato), alla libertà d'impresa. Spesso, per di più, con riguardo ad attività la cui natura rende la qualificazione giuridica di esse non pacifica (si pensi alla pubblicità, a cavallo fra libertà di manifestazione del pensiero e libertà d'impresa; o alla propaganda che, anche a seconda degli strumenti che utilizza, sembra rientrare o no nell'area della libera manifestazione del pensiero).

Una legislazione elettorale di contorno, dunque, quanto più è penetrante, tanto più interferisce su una molteplicità di valori costituzionali a fronte dei quali l'unico possibile approccio è quello indicato dalla giurisprudenza costituzionale, che impone al legislatore di assicurare un'armonica composizione di tali valori, attraverso il loro reciproco coordinamento in modo che l'eventuale compressione di uno di essi corrisponda a ragioni effettive e sia assistita da un necessario rapporto di «congruità e proporzionalità» rispetto al fine perseguito (in un quadro di «bilanciamento» fra valori costituzionali diversi). Ma, se non è impossibile legiferare sulla base di tali criteri quando si hanno da contemperare valori come la libertà di voto e la libertà di impresa, diverso è il caso quando si tratta di contemperare l'obiettivo della parità dei soggetti in competizione o il rispetto dell'autonomo convincimento dell'elettore con la libertà di manifestazione del pensiero: tanto più con riferimento a un procedimento, quello appunto finalizzato al voto, che costituisce la manifestazione più alta del confronto di opinioni.

Se le cose stanno così in termini generali, si capisce la varietà di soluzioni individuate dal legislatore e dettate dalla giurisprudenza costituzionale nei diversi ordinamenti.

$\mathrm{Si}$ pensi, per esempio, alla giurisprudenza della Corte Suprema americana che ha distinto il limite ai contributi elettorali (non incostituzionale), dal tetto alle spese elettorali (incostituzionale per violazione del Primo Emendamento) (v. la decisione Buckley vs. Valeo 424 US 1 (1976) largamente citata in Stone et al. 1991, 1312 ss., ed anche Gobbo 1991 e Gobbo 1994): mentre ha ammesso norme che, per incentivare la rinuncia a contri- 
buti illimitati, prevedono l'assegnazione ai candidati di contributi pubblici a condizione che accettino volontariamente un tetto per quelli privati. $\mathrm{O}$, in Germania, alla giurisprudenza della Corte di Karlsruhe che prima ha detto no al generico finanziamento dei partiti ammettendo il finanziamento delle campagne elettorali in quanto attività direttamente tutelate dalla Costituzione, poi, preso atto della tendenza del legislatore ad interpretarlo troppo estensivamente, ha imposto una nuova legislazione priva del c.d. contributo perequativo (volto ad accrescere le risorse dei partiti con meno autofinanziamento) ed ha anzi riconosciuto proprio nell'autofinanziamento di ciascun partito il limite oltre il quale non sono ammessi contributi pubblici, negando altresì legittimità anche alle deduzioni dall'imponibile (considerate sperequative ai danni degli elettori con reddito inferiore, sent. 19.7.1966 e sent. 9.4.1992). Quanto ai media essa ha discriminato fra mezzi di comunicazione di massa, ammettendo limiti alla pubblicità radiotelevisiva, ma non a quella sulla stampa (in altre parole riconoscendo che nel caso della radiotv la Chancengleichbeit fa premio sulla più ampia libertà dell'editore di pubblicare ciò che meglio crede, mentre l'opposto vale per la stampa) (Rizzoni 1994). Nel Regno Unito, dove la disciplina delle campagne elettorali è contenuta nella parte seconda del Representation of the People Act del 1983, si ritrovano tetti alla spesa dei singoli candidati, ma nessun tetto alla spesa dei partiti; la pubblicità politica sulla televisione è vietata, quella sui giornali ammessa.

La casistica comparata potrebbe continuare. I cenni fatti confermano che dal quadro comparato emergono soluzioni variegate e un'evidente difficoltà di individuare ragionevoli punti d'equilibrio fra valori diversi ma degni di protezione costituzionale. Nel caso italiano, tuttavia, il ritardo nell'affrontare organicamente la materia da parte del legislatore, appare soprattutto conseguenza della accentuata e tradizionale ritrosia del ceto politico italiano nei confronti di qualsiasi disciplina volta a introdurre limitazioni alla «sovranità di fatto» del partito politico e dei suoi esponenti².

Ciò contribuisce a spiegare perché, se si prescinde da alcu-

2 Sorte analoga, del resto, è sempre toccata a materia strettamente connessa: quella appunto della disciplina quadro dei partiti, ipotesi che non ha mai fatto passi avanti sicché i partiti restano da noi associazioni non riconosciute, pur finanziate dallo Stato con contributi annuali per ben 20 anni (1974-1993). 
ne proposte isolate ${ }^{3}$, non c'è mai stata in Italia fino al 1993 una disciplina unitaria dell'attività politica in vista delle elezioni: unica normativa specifica essendo la legge 4 aprile 1956, n. 212 (modificata in particolare dalla legge 24 aprile 1975, n. 130), la quale disciplinava «l'affissione di stampati, giornali murali od altri e di manifesti di propaganda» e poco altro ${ }^{4}$. Di qui la pressoché unanime denuncia di carenze e inadeguatezze di una legislazione che, per il resto, prevedeva solo la limitazione dell'uso di altoparlanti montati su vetture e la proibizione del lancio di volantini (legge 24 aprile 1975, n. 130, artt. 4 e 7), la disciplina della propaganda elettorale sui mezzi radiotelevisivi pubblici (legge 14 aprile 1975, n. 103, art. 4) e il divieto di propaganda e pubblicità sulle emittenti radiotelevisive private dal sabato prima del voto alla chiusura dei seggi (decreto legge 6 dicembre 1984 , n. 807 , convertito con modificazioni dalla legge 4 febbraio 1985 , n. 10$)^{5}$.

Non sorprende che la legislatura nella quale ciò che prima sembrava impossibile è divenuto non solo fattibile, ma addirittura obbligatorio, in ottemperanza all'espressa volontà costituente manifestata dagli elettori per via referendaria, ha prodotto, insieme alle nuove leggi elettorali per comuni, province e Parlamento, anche la nuova disciplina generale delle campagne elettorali, alla quale questo scritto è dedicato.

In effetti qualche tentativo, senza esito, si era già registrato nel corso della Decima legislatura, durante la quale in seno alla Prima Commissione della Camera si era addivenuti a un testo poi decaduto con la legislatura (per un'attenta analisi, si veda Troccoli 1992). Esso era in parte ispirato al modello francese e costituirà un punto di riferimento per i più fruttuosi tentativi dell'Undecima legislatura.

Proprio nel corso di questa, già alla Commissione parlamen-

${ }^{3}$ Segnalo quelle di Piero Calamandrei, di Costantino Mortati (1945), di Luigi Sturzo (1958), del deputato Fiori (1980), del deputato Valdo Spini (1984), lo schema del Pri (1965) mai presentato in Parlamento; lo schema del Club Turati (1968); lo schema del Movimento di opinione pubblica (1971): si ritrovano tutti in D'Antonio e Negri 1983, 609 ss., ad eccezione del progetto Spini, per il quale si veda invece Di Pace 1991.

${ }^{4}$ Lo fa in modo tanto minuzioso quanto, a ben vedere, «sproporzionato alla effettiva incidenza del mezzo» (Cheli 1981, 247).

5 $\mathrm{Di}$ «episodicità e disorganicità» e di «gravi carenze» parlano tutti coloro che hanno affrontato la materia (si possono leggere, in proposito, recentemente Lanchester 1988, specie 81 ss.; Romano 1991: Gobbo 1991; ma anche la più classica manualistica, da Mortati 1975 a Barile 1991). 
tare per le riforme istituzionali, in seno al «Comitato leggi elettorali» (relatore, nella prima fase, il sen. Salvi) era stata sottolineata l'esigenza che la nuova legislazione elettorale contenesse anche una nuova disciplina delle campagne elettorali; un altro tentativo era stato condotto al Senato, relatore il sen. Covatta, ma era stato travolto dalle polemiche quando il governo Amato aveva cercato di far proprie per decreto-legge alcune delle più delicate e controverse disposizioni di quel testo ed era stato costretto a rinunciarvi. Negli stessi giorni, tuttavia, le Camere erano riuscite a varare, in tempo per evitare il referendum, la nuova legge elettorale locale (legge 25 marzo 1993, n. 81), tre articoli della quale (gli artt. 28, 29 e 30, cioè l'intero Capo III) integravano la precedente frammentaria disciplina delle campagne elettorali con riferimento, naturalmente, alle sole elezioni amministrative. Si trattava di un intervento settoriale, approssimativo, ma ragionevolmente completo. Anch'esso giudicato affrettato (e probabilmente lo fu), costituisce per i suoi contenuti una sorta di «prototipo» in vista di una futura e più organica normazione (come osserva Gianluca Gardini, in Barbera 1993, 159-198), che individuava un nuovo equilibrio fra i diversi valori costituzionali in gioco.

Successivamente, a seguito di intese informali intervenute fra i Presidenti delle due Camere, fu il relatore della legge elettorale per il Senato, sen. Salvi, a inserire nello stesso testo unificato accanto al nuovo sistema elettorale una serie di norme di contorno relative alle campagne elettorali. Nel frattempo il referendum del 18 aprile 1993 si era risolto con l'abrogazione di quelle parti della legge 2 maggio 1974, n. 195 che prevedevano il contributo ordinario annuale ai partiti (artt. 3, 9). Sopravvivevano invece $\mathrm{i}$ contributi per il rimborso delle spese elettorali: la nuova disciplina delle campagne elettorali (sulla urgenza della quale l'accordo era unanime), si presentava come l'occasione propizia per rivedere, alla luce della legislazione elettorale in approvazione, anche il meccanismo di assegnazione e, soprattutto, l'importo di tale rimborso.

Stralciata dal resto della proposta in materia elettorale, essa fu approvata in prima lettura dal Senato il $1^{\circ}$ luglio 1993. Alla Camera veniva prima abbinata alla riforma elettorale (quella relativa all'elezione della Camera, ovviamente), poi finiva di nuovo stralciata a causa di contrasti su punti qualificanti, quali $(a)$ la richiesta di integrare il testo per farne una legge organica di disciplina delle campagne elettorali, applicabile non solo in oc- 
casione delle elezioni politiche ma anche in occasione degli altri tipi di elezione; $(b)$ il divieto di sondaggi; $(c)$ i contributi per le spese elettorali: nella dimensione e nei criteri di assegnazione; (d) i limiti alle spese non solo dei candidati ma anche dei parti$\mathrm{ti}$; infine, $(e)$ riemergeva prepotente la questione dell'apparato sanzionatorio per illeciti che i più non volevano di rilevanza penale ma sanzionabili con pene pecuniarie amministrative: si riproponeva la polemica sui «colpi di spugna» e ciò spiega la lentezza dell'iter e l'elaborazione di un altro testo, ancora più lontano da quello approvato dal Senato.

La legge 515/93 contiene dunque una disciplina organica delle campagne elettorali e tocca, in pratica, tutti gli aspetti rilevanti di una molteplice serie di attività accomunate dal periodo in cui si situano (dall'indizione dei comizi elettorali ovvero dal trentesimo giorno prima del voto) e dal fatto di essere finalizzate a influenzare le scelte degli elettori pro e contro uno o più candidati, una o più liste di candidati. Essa conferma l'inversione di tendenza, frutto del radicale processo di trasformazione politico-istituzionale in corso rispetto alla precedente tradizione di eccessivo self restraint legislativo nei confronti di processi politici che si volevano totalmente delegati ai partiti.

Gli argomenti che la legge affronta possono essere raggruppati nel modo che segue:

a) disciplina dell'accesso e della pubblicità sui mezzi di informazione (artt. 1, 2, 8, 15);

b) disciplina della propaganda elettorale nelle altre diverse forme (artt. 3, 5, 15);

c) disciplina dei sondaggi demoscopici (artt. 6, 15);

d) disciplina delle spese elettorali dei candidati (artt. 7, 8, $11,12,14,15)$, e dei partiti o movimenti (artt. 10, 11, 12, 15);

e) disciplina dei contributi pubblici per le spese elettorali (artt. 9, 15, 16); nonché previsione di una serie di agevolazioni a vantaggio di candidati e liste (artt. 17, 18, 19);

f) disciplina degli organi di controllo e del loro funzionamento (artt. 12, 13, 14);

g) individuazione dell'ambito di applicazione (rispetto ai diversi tipi di elezioni) degli artt. da 1 a 19 della legge stessa (art. $20)^{6}$.

${ }^{6}$ L'ambito di applicazione della legge ai diversi tipi di elezione è stabilito dall'art. 20 della legge 515/1993, una volta che il legislatore si è orientato verso una disciplina 


\section{L'accesso ai media}

La nuova disciplina dell'accesso ai mezzi di informazione di massa ha finito con il costituire, con particolare riferimento al settore radiotelevisivo, il tema di gran lunga più evocato non solo in margine all'applicazione della legge 515/93, ma addirittura nell'intera campagna elettorale della primavera 1994. Sarebbe stato sorprendente che accadesse altrimenti: basti tenere conto $(a)$ del contesto generale e, in particolare, dell'assetto del sistema radiotelevisivo italiano sancito dalla legge 6 agosto 1990, n. 223 (c.d. «Mammì); (b) dello schieramento delle forze che si contendevano il successo nel quadro del nuovo sistema elettorale a prevalenza maggioritaria, soprattutto dopo che l'azionista di controllo di gran parte del settore televisivo privato aveva annunciato la decisione di partecipare alle elezioni come candidato e, anzi, come potenziale leader di una futura maggioranza; $(c)$ della consolidata e pressoché incontrastata ten-

almeno tendenzialmente organica. Per una sintesi visiva propongo lo schema che segue:

Articolo legge 515/93 -

Elez. Politiche Europee Regionali Locali

\begin{tabular}{|c|c|c|c|c|c|}
\hline * 1 * & accesso media & SI & SI & SI & SI \\
\hline * 2 * & divieto pubblicità media & SI & SI & SI & [NO] \\
\hline * 3 * & altre forme di propaganda & SI & SI & SI & [NO] \\
\hline * 4 * & comunicaz. sui collegi & SI & {$[\mathrm{SI}]$} & [SI] & NO \\
\hline * 5 * & divieto propaganda ist. & SI & SI & SI & [NO] \\
\hline * 6 * & limiti ai sondaggi & SI & SI & SI & SI \\
\hline * 7 * & limiti spese candidati & SI & NO & NO & NO \\
\hline * 8 * & comunicaz. dai media & SI & NO & NO & $\mathrm{NO}$ \\
\hline * 9 * & contributo spese elett. & SI & SI & [NO] & [NO] \\
\hline * 10 * & limiti spese partiti & SI & NO & NO & NO \\
\hline * 11 * & tipologia spese elettorali & SI & SI & SI & SI \\
\hline * 12 * & pubblicità spese partiti & SI & SI & NO & [NO] \\
\hline * 13 * & collegio garanzia elettor. & SI & NO & NO & NO \\
\hline * 14 * & pubblicità spese candidati & SI & NO & NO & NO \\
\hline * 15 * & sanzioni & SI & {$[\mathrm{SI}]$} & [SI] & [SI] \\
\hline * 16 * & finanziamento contributi & SI & SI & NO & NO \\
\hline * 17 * & agevolazioni postali & SI & SI & SI & SI \\
\hline * 18 * & agevolazioni fiscali & SI & SI & SI & SI \\
\hline * 19 * & sedi comunali a disposiz. & SI & SI & SI & SI \\
\hline * 20 * & applicazione normativa & {$[\mathrm{NO}]$} & SI & SI & SI \\
\hline
\end{tabular}

Il [NO] fra parentesi quadra indica che la disciplina della materia non è nella 515/ $1993 \mathrm{ma}$ in altra legge; per le sanzioni i [SI] fra parentesi quadra indicano che solo alcuni commi del lunghissimo art. 15 si applicano ad elezioni diverse da quelle politiche; l'art. 20 è quello che estende parte della 515/1993 agli altri tipi di elezione (dunque non si applica per definizione alle elezioni politiche). 
denza a sopravvalutare (direi a mitizzare) l'influenza dei media sulle scelte elettorali.

Il regime precedente, invero, non prevedeva alcuna garanzia al di fuori del servizio pubblico: nell'ambito del quale, sin dai primi anni Sessanta (dopo la sentenza n. 59/1960 della Corte costituzionale) e poi più organicamente in ragione della disciplina di cui alla legge 103/1975 (la «riforma Rai»), condizioni di giuridica parità di trattamento erano assicurate attraverso il sistema delle c.d. «tribune» direttamente gestite dalla Commissione parlamentare di indirizzo e di vigilanza sul servizio radiotelevisivo (d'ora in poi «la Commissione parlamentare»); alle direttive e alla vigilanza della stessa Commissione (dunque della sua maggioranza) era affidata ogni garanzia per quel che riguarda il resto delle trasmissioni del servizio pubblico (con esiti che suscitavano periodiche polemiche in ordine alla propaganda che in forma surrettizia questa o quella rete Rai, il cui personale era apertamente «lottizzato» fra i partiti, andava facendo).

$\mathrm{Ma}$, se ciò valeva per la Rai, per il settore privato le uniche norme erano quelle contenute nel decreto $807 / 1984$, del quale si è già detto (divieto di propaganda dalla mezzanotte del venerdì prima del voto alla chiusura dei seggi). Per il resto, tutto era affidato (dopo che proposte di disciplina della propaganda politica erano state espressamente accantonate nel corso dell'iter legislativo), ai generici «principi fondamentali» dell'art. 1.2 della «Mammì (dove si parla di «pluralismo, obiettività, completezza e imparzialità dell'informazione» e di «apertura alle diverse opinioni, tendenze politiche, sociali, culturali e religiose...»); ai limiti generali alla pubblicità di cui all'art. 8 della stessa legge (che per esempio vietano la c.d. «pubblicità subliminale» e che pongono i noti tetti percentuali); alla disciplina del diritto di rettifica (art. 10). Infine, a interpretazioni attente e rigorose della normativa in materia di finanziamento dei partiti (ex legge 195/1974, come modificata dalla legge 659/1981). In sintesi: le garanzie della parità dei soggetti in competizione erano limitate e parziali nell'ambito del servizio pubblico, inesistenti nell'ambito del settore privato ${ }^{7}$.

L'art. 1 della legge 515/93 detta le norme di principio e di-

7 Si può citare la sentenza 13 giugno 1989 del pretore di Roma nella vertenza Democrazia Proletaria vs. Fininvest nella quale il giudice affermò che nessuna norma impone a un soggetto privato l'obbligo di estendere lo stesso trattamento nei confronti di tutti i partiti partecipanti alle elezioni. 
sciplina le modalità concrete (e i soggetti) che valgano a garantire appunto condizioni di «parità nei limiti del possibile» (così il ministro Elia, v. Atti Camera, Res. sten. 16 ottobre 1993, p. 31), secondo uno schema, nel quale:

- destinatari degli obblighi di cui all'articolo sono la concessionaria del servizio pubblico (a); gli editori di quotidiani e periodici $(b)$; i titolari di concessioni e di autorizzazioni radiotelevisive in ambito nazionale o locale, «nonché tutti coloro che esercitano in qualunque ambito attività di diffusione radiotelevisiva» $(c)$ : per ciascuno di essi la legge prevede una specifica e differenziata disciplina;

- attività soggette agli obblighi sono: rubriche elettorali, servizi o programmi di informazione elettorale $(a)$; spazi di propaganda elettorale $(b)$ (diversi dalle inserzioni pubblicitarie e dagli spot radiotelevisivi, di cui si occupa l'art. 2 della 515/93: anch'essa propaganda elettorale ma di «formato» diverso cui la legge dà una valutazione giuridica diversa); trasmissioni informative riconducibili a una specifica testata giornalistica registrata ai sensi di legge $(c)$; altre trasmissioni $(d)$;

- autorità cui è demandato il controllo sull'applicazione delle disposizioni sono: la Commissione parlamentare (a); il Garante per la radiodiffusione e l'editoria $(b)$; i Comitati regionali per i servizi radiotelevisivi $(c)$.

Pertanto: per quel che riguarda la Rai, in coerenza col regime fino ad ora differenziato che la caratterizza rispetto alle emittenti private, la legge conferma sostanzialmente il regime di rapporti preesistente: per i c.d. spazi di propaganda la Rai deve sottostare alle prescrizioni della Commissione parlamentare, e il principio da applicare è quello della parità fra liste e candidati; per le trasmissioni elettorali vere e proprie (tipo «tribune»), invece, la Rai è mera esecutrice di quanto dispone la Commissione che le «disciplina direttamente» (sotto questo aspetto, destinataria dell'obbligo di garantire parità di trattamento, completezza e imparzialità è la Commissione prima che la Rai). La Commissione deve dettare le sue prescrizioni alla Rai entro 5 giorni dall'indizione dei comizi elettorali. Il resto dell'universo delle trasmissioni radiotelevisive della Rai ricade sotto la disciplina generale del settore: la quale prevede per specifici soggetti («candidati, esponenti di partiti e movimenti politici, membri del Governo, delle giunte e consigli regionali e degli enti locali») il divieto di presenza in tutte le trasmissioni (art. 1.5) e una presenza limitata «esclusivamente alla esigenza di assicurare la 
completezza e l'imparzialità dell'informazione» nelle trasmissioni che possiamo definire «giornalistiche», nel senso definito dallo stesso comma 5: quelle «riconducibili alla responsabilità di una specifica testata» e dunque d'un direttore responsabile. Tali disposizioni si applicano dal trentesimo giorno prima del voto.

Per quel che riguarda le emittenti private, la decisione di mettere in onda trasmissioni di propaganda elettorale (a titolo gratuito oppure a pagamento) resta nell'autonomia di ciascun soggetto: se però l'emittente decide di farne, deve assicurare la parità di accesso a tutti i candidati, liste, gruppi di candidati, partiti o movimenti, secondo modalità quadro che (art. 1.3) sono dettate dal Garante per la radiodiffusione e l'editoria; di ciò ciascuna emittente deve dare informazione sul mezzo stesso prima del $30^{\circ}$ giorno dal voto; se l'emittente intende poi effettuare programmi di informazione elettorale (genere «tribune») deve anche in tal caso garantire la parità di trattamento a tutti i soggetti in competizione. Anche alle emittenti private si applicano le disposizioni relative alle «presenze» nelle trasmissioni giornalistiche e nel resto della programmazione.

Quanto, infine, agli editori di quotidiani e di periodici essi sono destinatari solo delle norme sulla propaganda elettorale e sulla pubblicità, ma non sono tenuti ad assicurare parità di trattamento negli articoli e servizi dedicati alle elezioni, né sono vincolati dalle limitazioni previste dall'art. 1.5 per la presenza dei soggetti interessati alle elezioni nelle trasmissioni informative e nel resto della programmazione radiotelevisiva.

Il Garante provvedeva il 26 gennaio 1994 a pubblicare il suo dettagliato «regolamento per la disciplina delle trasmissioni di propaganda elettorale»: un testo di 25 articoli, nel quale vengono meticolosamente dettate le regole (distinte fra stampa periodica e quotidiana, titolo I; e radiodiffusione sonora e televisiva, titolo II) per assicurare (i) la pubblicità delle condizioni di accesso alle trasmissioni di propaganda elettorale; (ii) l'effettiva parità di trattamento dei diversi soggetti (per esempio prevedendo complessi meccanismi volti a ridurre pro quota gli spazi eventualmente messi in vendita per far sì che nessun soggetto sia escluso per il solo fatto di essersi altri soggetti accaparrati tutti gli spazi disponibili); (iii) le modalità di determinazione delle tariffe; (iv) la riconoscibilità dei messaggi di propaganda; (v) l'esatta individuazione delle forme di propaganda vietate (la «pubblicità»: su questo v. dopo); (vi) le regole generali per i programmi e i servizi informativi (valide solo per le emittenti ra- 
diotelevisive, con l'espresso riconoscimento della libertà di commento e di critica, nell'ambito di una «chiara distinzione fra informazione e opinione», art. 16.3 del regolamento); (vii) il prolungamento, rispetto a quanto previsto dalla «legge Mammì, del periodo di conservazione delle registrazioni delle trasmissioni (esteso al termine delle procedure di controllo delle spese elettorali di cui alla legge 515/93); (viii) le funzioni attribuite ai Comitati regionali per i servizi radiotelevisivi.

Uniforme, invece, la disciplina della «pubblicità». Essa rientra nella ampia definizione di «propaganda elettorale» data dalla Corte di cassazione: «ogni attività inerente direttamente o indirettamente alla competizione elettorale e tendente, come scopo mediato o immediato, ad acquisire voti o a sottrarne agli avversari» (sent. 30 novembre 1972, in «Giur. pen.» 1973, II, 345), ma si distingue, nelle intenzioni del legislatore, dalle attività di propaganda di cui all'art. 1 della legge, per le modalità di esercizio («inserzioni pubblicitarie», «spot pubblicitari e ogni altra forma di trasmissione pubblicitaria radiotelevisiva»), al punto da giustificare un (teorico) secco divieto (sulla falsa riga dell'art. 29.1 della legge 81/93, riguardante gli enti locali).

Il punto è delicato: perché è chiaro che distinguere nettamente l'un tipo di propaganda dall'altro, non è cosa pacifica (così come distinguere la propaganda dall'informazione) (Lanchester 1988 e 1994). Ciò è tanto vero che, come si accennava poco $\mathrm{fa}$, il Garante nei suoi regolamenti si è sentito in dovere di suggerire una più esatta definizione della propaganda vietata in quanto «confezionata» in forma di pubblicità: secondo l'art. 6 del suo regolamento, si considerano inserzioni pubblicitarie vietate ai sensi della 515/93 «le pubblicazioni esclusivamente di slogan positivi o negativi, di foto o disegni, di inviti al voto non accompagnati da adeguata, ancorché succinta, presentazione politica di candidati e/o di programmi e/o di linee ovvero da una critica motivata nei confronti dei competitori» (per la stampa); mentre per la radiotelevisione, secondo l'art. 15, sono vietate $(a)$ «le trasmissioni contenenti esclusivamente elementi di spettacolarizzazione, scene artificiosamente accattivanti anche per la non genuinità di eventuali prospettazioni informative, slogan, inviti al voto non accompagnati da un'adeguata - ancorché succinta - presentazione politica di candidati e/o di programmi e/o di linee» nonché $(b)$ «le trasmissioni che usano, attraverso elementi atti a destare rifiuto, le stesse tecniche di suggestione indicate sotto la lettera a) per dare esclusivamente 
un'immagine negativa dei competitori...». Rinunciando ad ogni tentazione di facile ironia, va detto che siamo di fronte a un coraggioso e, in fondo, encomiabile sforzo di definire l'indefinibile, un esempio di alto equilibrismo normativo, destinato ad aprire un contenzioso senza fine e di incerta costituzionalità.

Come è stato osservato, dal momento che la propaganda (anche sui giornali, anche sulla radiotelevisione) non è proibita se non nella forma di inserzioni e spot, non sarà il contenimento delle spese e dunque, indirettamente, la parità delle chances, né la volontà di moralizzare le campagne elettorali la giustificazione di tale difforme trattamento: unico possibile aggancio resta la motivazione (assai discussa in dottrina) (Cerri 1985), con la quale la Corte costituzionale sancì la legittimità costituzionale del divieto di propaganda sonora e luminosa disposto dalla legge 130/1975 (v. sent. 138/1985, fra l'altro in «Giur. cost.» 1985, I, p. 987), giustificandolo appunto con il diritto dei cittadini a non subire «un'invasione incontrollata della propria sfera di vita privata da parte di manifestazioni propagandistiche troppo aggressive». Principio in sé ragionevole ma di applicazione assai complessa e necessariamente troppo discrezionale ${ }^{8}$.

Del resto ho definito «teorico» il divieto di cui si discute: soprattutto grazie alle deroghe contenute, nello stesso art. 2 della 515/93 (anche qui secondo il modello della 81/93), deroghe che finiscono con il rendere un gioco da ragazzi qualsiasi aggiramento del divieto stesso: nel quale non rientrano né gli annunci di dibattiti, tavole rotonde, conferenze, eccetera; né la presentazione di programmi e di candidati; né i confronti fra più candidati. L'impressione è di trovarsi davanti a una normamanifesto di incerta costituzionalità, d'impossibile e forse inutile applicazione, una sorta di concessione gratuita e senza conseguenze concrete a quel tanto di moralismo anti-mercato che, nonostante tutto, sembra ancora riscuotere consensi?.

8 Alla sensibilità di chi scrive, non parrebbe davvero, per esempio, che gli spot di pubblicità elettorale costituiscano forme di propaganda in sé aggressive, tanto più per una pubblica opinione abituata ormai da anni a ben altre forme di martellamento pubblicitario. Di più: si sentirebbe di contestare energicamente che le inserzioni sui giornali abbiano alcunché di aggressivo. Sembrerebbe, anzi, che si tratti di forme di propaganda particolarmente «fredde»: e per il supporto tecnico e per momenti in cui questo viene letto. Ma tant'è.

9 Non questa osservazione (ma altre...) avrebbe meritato invece una normativa che avesse vietato qualsiasi forma di propaganda elettorale sui media come fa per esempio la legislazione inglese (v. Gardini 1994). Sul punto si segnala criticamente la diffusa con- 
Al di là delle valutazioni già formulate in tema di pubblicità, aggiungo che, in termini generali, norme vincolistiche del genere di quelle illustrate sono comunque destinate ad incontrare difficoltà di applicazione e a prestare il fianco a efficaci tentativi di aggiramento: per esempio, è possibile da parte dell'editore o del titolare di concessione radiotelevisiva favorire questo o quel soggetto presente nella competizione elettorale elaborando «pacchetti» di spazi di propaganda elettorale i quali, pur offerti a parità di condizioni a tutti i soggetti in competizione, siano «tagliati» su misura a vantaggio di uno specifico soggetto.

Al tempo stesso appare comprensibile nelle sue motivazioni, e nondimeno presuntuosa la pretesa di assicurare per legge una parità di trattamento nelle trasmissioni di carattere giornalistico: mentre la norma limitatrice delle presenze può avere una qualche applicazione (ma chi apprezza la «completezza e imparzialità» della scelta?), risulta di fatto impossibile conciliare parità di trattamento (almeno se intesa per così dire «aritmeticamente»: ma su questo tornerò) e libero esercizio della professione giornalistica: come l'ordine nazionale dei giornalisti ha sostenuto in un comunicato del venerdì precedente il voto, «non ha senso che il legislatore preveda per legge l'obiettività» (Ansa, 25 marzo 1994).

Una discussione sul punto porterebbe lontano. Certo è che, sotto la pressione di taluni partecipanti alla campagna elettorale e su indiretto e autorevole invito dei Presidenti delle Camere, le principali reti (Rai e Fininvest) sottoscrivevano, il 18 febbraio 1994, un «accordo sul comportamento da tenere nelle trasmissioni durante il periodo elettorale» che costituisce di fatto l'equivalente di quei «codici di stile» tipici delle grandi aziende (anche informative) anglosassoni ${ }^{10}$ e il Garante, in un appello

vinzione secondo la quale «informare» sarebbe cosa buona, «emozionare» cosa cattiva (Rodriguez 1994, 139). Quanto sono andato esponendo in ordine agli artt. 1 e 2 della legge non si applica agli organi ufficiali di stampa e radiofonici dei partiti.

${ }_{10}$ L'accordo dettava regole precise per ogni tipo di trasmissione informativa che trattasse «tematiche di natura politico-elettorale» nonché per quelle che «prevedendo la presenza a qualsiasi livello dei soggetti competitori, siano comunque da considerare... come trasmissioni di propaganda elettorale...». Si tratta del testo in cui si dice ai conduttori come fare le domande («in termini chiari, non faziosi né tendenziosi»); quanto tempo dare a tutti i partecipanti $\mathrm{e}$ in che modo consentire a ciascuno di rispondere («senza inutili interruzioni nel rigoroso rispetto del tempo preventivamente assegnato»); come evitare «qualsiasi condizionamento e artificio, per finalità di spettacolarizzazione o di audience»; come disciplinare la eventuale presenza del «pubblico» che (quante vol- 
informale, richiamava $\mathrm{i}$ conduttori a fare attenzione agli «sguardi e gesti» coi quali si rivolgevano agli eventuali ospiti in trasmissione: anch'essi potevano violare la parità.

Tutte cose giuste, ma note, si dovrebbe presumere, al giornalista ligio alla propria professione e rispettoso della propria dignità. Tutte cose, peraltro, che se si è sentita la necessità di richiamarle, venivano percepite da taluni come carenze oggettive e ignorate, ahimè, da altri. In sintesi: ecco altre disposizioni manifesto buone come occasione, a ben vedere nient'affatto superflua, per indurre a un più maturo modo di fare informazione. Riguardo alle quali, peraltro, va detto che il loro tradursi in comportamenti concreti è destinato a dipendere più da forme di controllo collettivo incrociato dei soggetti in competizione e degli stessi media e da una maturazione complessiva del corpo sociale, che da controlli e sanzioni di carattere strettamente giuridico. Il moltiplicarsi in campagna elettorale dei vari «osservatori» (in un caso almeno finanziati dalle stesse emittenti) sembra suffragare questa convinzione.

\section{Le sanzioni}

Quanto, appunto, all'apparato di controllo, abbiamo già visto che $\mathrm{i}$ relativi compiti sono affidati dalla legge per alcuni aspetti alla Commissione parlamentare (la quale peraltro non ha praticamente mai funzionato durante la campagna elettorale a causa della cronica mancanza del numero legale) e, per quelli di maggior rilievo, al Garante per l'informazione e l'editoria, coadiuvato, ai fini del controllo, dai Comitati regionali per i servizi radiotelevisivi. $\grave{E}$ una delle parti maggiormente criticate della legge 515/93, così come non è passato indenne da furibonde polemiche il Garante stesso cui la sua applicazione era affidata, accusato (ma tale critica non è coerente con l'altra) di presunta timidezza nell'esercizio dei poteri conferitigli. Alle accuse il $\mathrm{Ga}$ rante ha risposto ribaltandole sull' «inadeguatezza, la carenza, la "superficialità" della legge» (v. Rapporto dell'Ufficio del Garante concernente il periodo elettorale del 6 maggio 1994, p. 6) e rivendicando, invece, le cose fatte: 85 «contestazioni con diffi- 
da» nel periodo elettorale; il regolamento di cui si è ampiamento detto; una serie di richiami di moral suasion, per lo più in direzione del rispetto della parità di trattamento nelle trasmissioni (non nell'offerta di propaganda o pubblicità a pagamento); una serie di chiarimenti scritti a singole emittenti, ai Comitati regionali, ai Circostel (Circoli per le costruzioni telegrafiche e telefoniche). Il Garante, infine, ha lamentato la carenza di mezzi a disposizione (ma il decreto-legge 23 febbraio 1994, n. 129, art. 4 gli attribuiva la possibilità di utilizzare anche la Guardia di finanza) ${ }^{11}$.

L'intero apparato sanzionatorio della legge 515/93 si ritrova nell'art. 15. Il comma 1 è quello che riguarda le sanzioni rispetto alle violazioni degli artt. 1 e 2 della legge. Si tratta (come negli altri casi) di sanzioni amministrative pecuniarie, affidate nella loro applicazione, in questo caso, appunto al Garante che può infliggerle anche ai candidati o ai partiti a vantaggio dei quali le violazioni siano state commesse (qualora ne sia accertata la corresponsabilità). Le procedure sono quelle di cui alla legge 689/81. Esse prevedono (a) un atto di contestazione e notifica; $(b)$ termini di 30 giorni per fornire giustificazioni; $(c)$ il diritto del destinatario della contestazione di farsi ascoltare; $(d)$ l'eventuale archiviazione o sanzione; $(e)$ il ricorso al Tribunale amministrativo regionale. Una procedura non rapidissima, quindi. Tuttavia, in campagna elettorale, il Garante può anche diffidare le emittenti a «ripristinare... non oltre tre giorni, le condizioni al cui rispetto sono tenuti per legge e per disposizione del Garante o della Commissione parlamentare...»: in caso di inottemperanza può ordinare la sospensione delle trasmissioni («sospensione dell'efficacia della concessione o dell'autorizzazione», in termini giuridici) per un periodo da 11 a 30 giorni. Nei casi più gravi, può proporre al Ministro la revoca della concessione o autorizzazione.

Il Garante spiega la sua presunta passività, oltre che con la solita limitatezza di mezzi, con nell'ordine: $(a)$ la lentezza dei Circostel e dei Comitati regionali nel segnalare le violazioni; $(b)$ i tempi delle successive verifiche; $(c)$ il fatto che le previste «sanzioni interdittive» impedirebbero «interventi immediati» in assenza di un vero e proprio «potere cautelare» (peraltro attri-

${ }^{11}$ Secondo un giornale, questa metteva a disposizione sua e dei collegi regionali di garanzia elettorale, sui quali v. dopo, ben 700 «fiamme gialle», v. l'«Unità» del 25 febbraio 1994. 
buitogli dal decreto legge 23 febbraio 1994, n. 129, art. 5 per le violazioni compiute da emittenti prive di concessione o autorizzazione»); (d) il fatto che la legge, nel prevedere una sospensione minima di ben 11 giorni, impedisce un'opportuna modulazione dell'intervento. Peraltro, pur cumulate tutte queste spiegazioni, il Garante afferma poi che «per quel che riflette le misure interdittive, l'Ufficio non ha ravvisato che sussistessero gli elementi oggettivi per applicarle...»(p. 4 del citato Rapporto). Par di capire, perciò, che al di là di ogni questione di mezzi giuridici e materiali a disposizione, egli ha comunque ritenuto che non si siano date le condizioni per interventi drastici come la sospensione delle trasmissioni.

\section{Il problema della parità}

Altrettanto delicato il tema della «parità» cui la legge fa riferimento. Al riguardo è stato più volte evocata la risoluzione 900 (1988) dell'Assemblea parlamentare del Consiglio d'Europa, relativa proprio all'accesso ai mezzi di comunicazione audiovisuale durante le campagne elettorali. In questo documento, peraltro, nel momento in cui si invitano i governi degli Stati membri a prendere provvedimenti per assicurare una «reale uguaglianza di opportunità... fra tutti i partiti politici...», si intende tale pari opportunità riferita alla «rappresentatività sociale e politica» dei partiti stessi, parametrando poi questa «essenzialmente» (non esclusivamente) ai «risultati ottenuti alle ultime elezioni legislative a suffragio universale» (esattamente come in Germania) (Rizzoni 1994).

Cito il documento solo per richiamare quanto è del resto ovvio: e cioè che espressioni come «parità», «parità di trattamento», «principio di parità» possono essere interpretate nel senso che nessuno può essere discriminato rispetto agli altri, ovvero nel senso che ciascun soggetto ha diritto non ad essere trattato secondo un uguale parametro, ma a vedersi assegnare (nel caso di specie) spazi uguali, tempi uguali, fasce orarie uguali (o equivalenti), eccetera. È questione antica che va ben al di là di ciò che si deve intendere per «pari opportunità» dei competitori in una campagna elettorale.

Anche volendo optare, secondo la tendenza emersa di recente nelle decisioni della Commissione parlamentare e del Garante, per una parità di trattamento intesa come effettiva uguale 
«esposizione», sul mezzo considerato, c.d. equal time (sia pure, come è stato specificato nell'accordo Rai-Fininvest citato, nel quadro non della singola trasmissione ma del complesso delle trasmissioni di una certa serie), non è che tutto si risolva: ed anzi, altri problemi insorgono. Ai fini delle presenze radiotelevisive, per esempio, di cosa tenere conto, stante la struttura della competizione elettorale del 1994: delle coalizioni in competizione oppure dei singoli soggetti all'interno di ciascuna coalizione? Nel primo caso, che trattamento riservare ad eventuali ulteriori soggetti in competizione? Nel secondo, come evitare di avvantaggiare le coalizioni costituite da un maggior numero di soggetti rispetto a quelle costituite da pochi soggetti? ${ }^{12,13}$.

Infine, emerge già dagli artt. 1 e 2 della 515/93 la questione (evocata fra l'altro anche dal Garante nel Rapporto citato) della durata della campagna elettorale $o$, più esattamente, dell'ambito temporale di applicazione delle disposizioni della nuova disciplina: altro aspetto criticatissimo della legge.

Se l'osservazione, secondo la quale la distinzione fra propaganda politica e propaganda elettorale si è fatta sempre più tenue (dal momento che le forze politiche sono protagoniste di una sorta di «campagna elettorale permanente») (v. anche Rodriguez 1994) non è nuova, si spiegano bene le ragioni in base alle quali, da noi, la disciplina della campagna elettorale ha trovato sin dalle origini applicazione limitata nel tempo. Va subito chiarito che non esiste nella nostra legislazione, né è mai esisti-

12 Ciò è quanto è effettivamente successo al Patto per l'Italia secondo quanto dimostra, fra le altre, l'indagine curata da Morcellini: la logica dei raggruppamenti «soprattutto nel momento in cui si adotti l'impostazione apparentemente neutra dell'equal time... si traduce infatti in una preponderante visibilità dei raggruppamenti composti da un maggior numero di partiti...» (Morcellini 1994, 15).

${ }^{13}$ Le indagini dei vari osservatori che si sono dedicati a seguire la campagna elettorale sui media («Mediamonitor» v. Morcellini 1994; ricerca curata da Rositi dell'Università di Pavia, dati pubblicati sui giornali del 25 marzo 1993; dati della società Canale Tre, cit. nel «Sole 24 ore» del 25 marzo 1993; dati della ricerca curata da Ricolfi, Dipartimento di Scienze sociali dell'Università di Torino, pubblicati sui giornali del $1^{\circ}$ giugno 1994) sembrano smentire le valutazioni più pessimistiche sull'andamento della campagna elettorale 1994 in radio e in tv e sugli effetti della legge 515/93. In particolare, la ricerca «Mediamonitor» sembra dimostrare: $(a)$ che la rappresentazione bilanciata degli schieramenti in campo prospettata dal sistema televisivo è stata sostanzialmente omologa a quella uscita dalle urne; $(b)$ che vi è stata una «straordinaria» somiglianza delle «quote di visibilità» dei diversi protagonisti sulle reti Rai e su quelle Fininvest; $(c)$ che «a nessun rassemblement è stata tappata la bocca», anche se $(d)$ i due oligopolisti hanno invece dimostrato meno imparzialità per quel che riguarda $\mathrm{i}$ partiti singoli $\mathrm{e} i$ leader (v. Morcellini 1994). 
ta, una «durata giuridica» della campagna elettorale: semplicemente le norme destinate a disciplinare la propaganda elettorale si è da sempre ritenuto che fossero materialmente applicabili solo dal giorno successivo a quello che il testo unico delle leggi elettorali (il fondamentale t.u. 30 marzo 1957, n. 361 con tutte le sue numerosissime modificazioni fino alle ultime del d.lgs. 20 dicembre 1993, n. 534) prevede come termine per la presentazione delle candidature, nel presupposto che solo da quel momento è possibile individuare i destinatari delle norme limitatrici della propaganda elettorale ovvero (dopo la 515/93) titolari del diritto alla parità d'accesso ai media ${ }^{14}$.

Ora, se si considera che, come già ampiamente sottolineato, siamo di fronte a disposizioni limitatrici rispetto a quello che è l'ordinario esercizio di diritti costituzionalmente protetti (ed anzi di quelli qualificati come inviolabili), non sorprende che il legislatore non si sia assunto la responsabilità di far slittare troppo indietro nel tempo tali misure restrittrici: tanto più se si considera la difficoltà e la potenziale incertezza nell'individuazione dei soggetti cui esattamente riferirla.

In effetti, sono state le vicende concrete d'una campagna elettorale per tanti aspetti inattesa a complicare le cose e ad esacerbare le polemiche: la presenza di un nuovo soggetto politico, strettamente legato all'oligopolio televisivo privato, il quale pienamente si avvantaggiava dei mezzi di cui si poteva presumere disponesse in condizioni di vantaggio (quanto meno fuori dalla efficacia delle norme sulla parità), induceva i più a ritenere che più opportuno sarebbe stato anticipare, magari fissandola al giorno successivo all'indizione dei comizi elettorali, la data dalla quale parte delle disposizioni della $515 / 93$ si dovessero applicare (e in particolare gli artt. 1 e 2 ). Al punto che, da varie parti, si sollecitava il Governo del tempo a intervenire sulla materia per decreto, modificando, su un punto così delicato, una legge pur approvata dal Parlamento solo 45 giorni prima. Saggiamente il Governo si asteneva dall'intervenire e il Ministro per i rap-

14 Infatti, le candidature vanno presentate entro le ore 20 del $34^{\circ}$ giomo prima del voto; entro il $33^{\circ}$ si pronuncia l'Ufficio centrale circoscrizionale; entro il $32^{\circ}$ l'Ufficio decide sugli eventuali ricorsi; entro il $31^{\circ}$ le Giunte comunali delimitano, ripartiscono fra i concorrenti e assegnano gli spazi da destinare alle affissioni di propaganda elettorale: ecco perché, infine, il $30^{\circ}$ giorno è stato individuato come quello entro il quale scatta l'applicazione delle varie disposizioni delle due leggi fondamentali della materia, la vecchia 212/1956 (con successive modificazioni) e la 515/93. 
porti col Parlamento Barile dichiarava, con rammarico, che il Governo non poteva cambiare le regole «a gioco avviato» (v. i giornali dell'8 febbraio 1994).

Del resto, la 515/1993 prevede una calcolata differenziazione dei tempi di applicazione delle proprie disposizioni, che scattano secondo questo schema al quale non si può negare una certa razionalità, pur con qualche incongruenza:

* dall'entrata in vigore della legge:

- obbligo di costituzione dei Collegi regionali di garanzia elettorale (v. dopo)

* dal giorno stesso dell'indizione dei comizi:

- obbligo per i comuni di mettere a disposizione i locali

* dal giorno dopo l'indizione dei comizi:

- raccolta dei fondi solo attraverso mandatario (v. dopo)

* entro 5 giorni dall'indizione dei comizi:

- obbligo per la Commissione parlamentare di dettare prescrizioni al servizio pubblico in ordine alla propaganda

* dal $60^{\circ}$ giorno prima del voto:

- divieto di propaganda istituzionale da parte di tutte le pubbliche amministrazioni (v. dopo)

* dal $30^{\circ}$ giorno prima del voto:

- norme sulla parità d'accesso su tutti i media

- norme sui limiti alle presenze in trasmissione di particolari soggetti

- divieto di propaganda mediante inserzioni e spot

- limiti alle altre forme di propaganda sulla base della legge 212/1956

- possibilità per candidati e liste di usufruire delle agevolazioni postali

* dal $15^{\circ}$ giorno prima del voto:

- divieto di diffusione di sondaggi sull'esito del voto

* dal giorno precedente il voto:

- divieto di (quasi) tutte le forme di propaganda.

Per concludere sulla disciplina dell'accesso e della pubblicità, il vero problema non è mai stato la disciplina della campagna elettorale, quanto l'assetto del sistema radiotelevisivo italiano: problema che non si risolve con una legge sulle campagne elettorali per buona che essa sia.

Ogni forma di propaganda elettorale diversa da quella che utilizza i media è disciplinata dall'art. 3 della legge 515/93, il quale innanzitutto fa rinvio (per le forme specifiche ivi regolate) alla legge 212/1956 con le sue successive modificazioni. 
È il comma 2 dell'art. 3 a generalizzare un'innovazione che aveva già trovato spazio (con le stesse parole) nel comma 3 dell'art. 29 della legge sulle elezioni amministrative: l'obbligo che ogni «materiale» di propaganda elettorale porti l'indicazione del «committente responsabile» (la disposizione si applica anche alla propaganda a mezzo stampa quotidiana e periodica e a mezzo radiotelevisione). Ad integrazione della disciplina del marzo 1993, tuttavia, il successivo comma 3 aggiunge l'importante obbligo, a carico di chi fornisce materiale o servizi «utilizzabili in qualunque forma a scopo di propaganda elettorale», di accertarsi che l'ordine sia fatto da persona che poi possa esserne chiamata a risponderne (i segretari amministrativi o delegati responsabili della propaganda per i partiti; i singoli candidati o i loro mandatari per i candidati individualmente considerati): in tal modo la legge assicura l'imputazione certa della spesa sostenuta a fronte dei tetti di cui ai successivi artt. 7 e 10. Si tratta di disposizioni assistite da sanzione pecuniaria amministrativa di lire da 1 a 50 milioni, a mente dell'art. 15.2: dunque di sicura efficacia deterrente rispetto al presumibile quadro di convenienze economiche di qualsiasi operatore.

Per comodità di esposizione riferiamo qui anche del divieto, contenuto nell'art. 5, per «tutte le pubbliche amministrazioni» (dunque: centrali e periferiche, statali, regionali, provinciali, comunali e quant'altro) di svolgere attività di propaganda «di qualsiasi genere, ancorché inerente alla loro attività istituzionale» sin dal trentesimo giorno antecedente l'inizio della campagna elettorale (dunque a partire dal $60^{\circ}$ giorno prima del voto). Fanno eccezione solo quelle attività di comunicazione istituzionale «indispensabili per l'efficace assolvimento delle funzioni proprie delle amministrazioni pubbliche» (per esempio l'annuncio del nuovo orario di apertura di un ufficio o della sospensione della fornitura di acqua potabile e simili). Siamo, con tutta evidenza, di fronte, a una norma che dev'essere considerata di diretta attuazione dell'art. 97 Cost. laddove questo afferma il principio dell'«imparzialità dell'amministrazione». La disposizione, $\mathrm{i}$ cui destinatari erano numerosi (e in molti casi male abituati), ha costituito una novità di un certo rilievo (a parte il precedente del comma 6 dell'art. 29 della 81/93) ${ }^{15}$. La sua applica-

15 Su sollecitazione dell'Associazione nazionale comuni d'Italia (Anci) il $1^{\circ}$ febbraio 1994 la Direzione centrale per i servizi elettorali del Ministero dell'interno dava un'interpretazione ragionevolmente restrittiva del divieto (dunque «larga» nei confronti 
zione sembra aver avuto effetti visibili nella campagna elettorale 1994 nella quale decine di manifestazioni e convegni sono stati effettivamente annullati o rinviati dalle pubbliche amministrazioni, nonostante che, come è stato subito osservato, non si rinvengano nella legge 515/93 sanzioni contro eventuali violazioni del precetto ${ }^{16}$.

\section{La disciplina dei sondaggi}

Un'assoluta novità, per l'ordinamento italiano, è costituita dalla disciplina dei sondaggi demoscopici di cui all'art. 6: altro complesso di disposizioni fra quelle che hanno suscitato interesse e discussioni talora accese soprattutto dacché l'utilizzo dei sondaggi è diventato molto diffuso acquistando una sua «centralità» nell'intera vicenda elettorale, non solo come strumenti di previsione, ma anche come mezzi per influenzare le scelte degli elettori, come essenziali tools per la definizione da parte dei soggetti in competizione delle proprie strategie di marketing politico, come strumenti, infine, per la successiva verifica di tali strategie.

In Italia la prima proposta è quella del 1984 fatta dal deputato Battistuzzi (v. AC 1026 - XI legislatura), cui molto si ispira la legge 515/1993. Nel testo della futura legge sulle campagne elettorali elaborato in prima lettura dal Senato si era previsto un drastico divieto di fare sondaggi (per i 30 giorni prima del voto). Il legislatore ha alla fine varato un compromesso fra le tendenze contrapposte, che si fonda sui seguenti punti:

- non è vietato fare sondaggi, ma solo «renderli pubblici o comunque diffonderli»;

- sono vietati non solo i sondaggi sull'esito del voto, ma anche quelli, più generali, sugli orientamenti politici degli elettori

delle iniziative delle amministrazioni pubbliche) nel senso che rientrerebbero nel divieto «solo le attività amministrative collegabili, direttamente o indirettamente, a qualsivoglia propaganda elettorale di candidati o liste...» (circolare 12/92 del 1 febbraio 1994, nel «Sole 24 Ore» del 2 febbraio 1994).

${ }^{16}$ Eventualmente, occorrerebbe rifarsi a norme generali, in effetti di dubbia applicabilità data la sproporzione fra violazione e «sanzione», in senso atecnico: per esempio nei confronti degli enti locali, potrebbe essere invocato l'art. 39 della legge 142/90 che prevede lo scioglimento dei consigli con decreto del Presidente della Repubblica su proposta del Ministro dell'interno in caso di «atti contrari alla costituzione o per gravi e persistenti violazioni di legge...». 
(il che allarga l'area di applicazione della norma in misura rilevante);

- il divieto vige nei 15 giorni prima del voto;

- in ogni caso, cioè indipendentemente da qualsiasi termine, la pubblicazione o diffusione (anche parziale) di sondaggi sulle elezioni politiche (non sulle altre) deve essere accompagnata da una serie di indicazioni che permettano una compiuta valutazione della qualità del sondaggio stesso ${ }^{17}$;

- le sanzioni contro le violazioni sono molto pesanti e sono state aggravate mediante decreto legge (d.1. 4 febbraio 1994, n. 88): se la violazione del divieto avviene fino all'inizio delle votazioni la sanzione amministrativa pecuniaria va da 100 a 1.000 milioni; se essa avviene a urne aperte, si applica oltre alla stessa sanzione amministrativa pecuniaria, la pena detentiva di cui all'art. 100 del T.U. 361/1956 (reato di turbativa elettorale): ci si riferisce agli exit-polls; se mancano le indicazioni relative alle caratteristiche del sondaggio la sanzione amministrativa pecuniaria va da 1 a 100 milioni;

- le sanzioni sono inflitte dal Garante, ad eccezione di quelle in caso di violazione del divieto a urne aperte, ovviamente di competenza del giudice ordinario.

Limitazioni nei confronti dei sondaggi sono ben conosciute al diritto comparato (si pensi al consueto modello francese, forse il più meticoloso nel disciplinare ogni aspetto del fenomeno, che prevede però un divieto di pubblicazione limitato alla settimana precedente il voto; al modello spagnolo, anch'esso alquanto dettagliato e con divieto limitato ai cinque giorni prima del voto; nulla si rinviene nella legislazione tedesca e inglese) (v. La legislazione elettorale 1994, 94-101). Nel quadro dell'ordinamento italiano, destinatari dell'obbligo di non diffondere l'esito di sondaggi elettorali nei 15 giorni del divieto sono sia i soggetti che realizzano il sondaggio, riguardo ai quali, come è stato osservato, la pubblicazione del sondaggio stesso non può ricondursi automaticamente nell'ambito dell'art. 21 Cost., ma forse, piuttosto nell'art. 41 Cost.; sia i soggetti committenti che ricevano, riservatamente, l'esito del sondaggio dal soggetto che l'ha realizzato (e in tal caso si rientra senz'altro in aree protette dall'art. 21 Cost.).

17 Esse coincidono in parte, $\mathrm{ma}$ in misura secondo $\mathrm{i}$ critici inadeguata, con i dettami del Codice di autoregolamentazione dell'European Society for Opinion and Marketing Research (Zanaroli 1994, 24). 
Come che sia, il valore che il legislatore, in questo caso, ha voluto tutelare prioritariamente è, di nuovo, l'autonoma formazione della scelta da parte dell'elettore, nella convinzione che essa debba essere difesa dalla forza suggestiva di interessate (o anche disinteressate) previsioni. La logica è la stessa che giustifica i limiti alla propaganda radiotelevisiva: ma resta pur sempre $\mathrm{da}$ chiedersi se la conoscenza delle previsioni formulate in base a sondaggi (purché ne sia in qualche modo garantita la relativa attendibilità o comunque la serietà nella raccolta ed elaborazione dei dati o, almeno, gli elementi per valutare l'una e l'altra) diminuisce o, al contrario, accresce la libertà di scelta dell'elettore. Ottimi argomenti possono essere spesi in entrambe le direzioni (è degna di esser tutelata come genuina una scelta fatta nell'ignoranza del rispettivo peso delle forze e delle candidature in campo, oppure è degna di essere tutelata una scelta fondata sul maggior numero possibile di informazioni, ivi comprese quelle sugli altrui orientamenti, in modo, per esempio, da favorire il c.d. «voto strategico» o «utile»?).

Il fatto è che, come per la radiotelevisione, siamo di fronte a un caso in cui il legislatore mostra di mitizzare certi strumenti attribuendo ad essi una potenzialità di condizionamento e di distorsione assolutamente sproporzionata: senza contare, poi, che, nel caso dei sondaggi, più sondaggi diversi dovrebbero alla fine neutralizzarsi (sul punto cfr. Zanaroli 1994).

\section{Le spese elettorali}

Accanto alle norme sull'accesso ai media, l'altro pilastro della legge 515/93 è il complesso di disposizioni volte a disciplinare le spese elettorali di candidati e forze politiche. Anche in questo caso, il legislatore non ha lesinato gli strumenti per limitare i costi della politica e assicurarne la trasparenza finanziaria.

Non è qui possibile soffermarsi su un iter legislativo rivelatore ed interessante. Dirò solo che la questione cruciale è stata se includere nella disciplina solo i candidati oppure anche le forze politiche (che ancora una volta tentavano di sottrarsi a una disciplina): la soluzione approvata le comprende, anche se trova come pendant l'introduzione nella legge d'un generoso aumento dei contributi per le spese elettorali.

Ecco i capisaldi della disciplina:

- è previsto un limite massimo delle spese elettorali di cia- 
scun candidato (art. 7.1); il tetto, calcolato su una base fissa di 80 milioni cui si aggiunge un tanto a cittadino residente, va da 90 a 120 milioni circa e sarà periodicamente rivalutato sulla base degli indici Istat dei prezzi all'ingrosso (art. 7.8); nel caso di candidatura doppia (circoscrizionale e uninominale in un collegio della stessa circoscrizione) il tetto è dato dall'importo più alto dei due previsti; nel caso di candidatura in più circoscrizioni diverse, invece, $\mathrm{i}$ singoli tetti si sommano (tale interpretazione non è pacifica perché sul punto la legge non è chiarissima: ma la logica del testo è evidente, impedire che un candidato, per fare propaganda allo stesso elettorato, possa godere di un tetto computato due volte ${ }^{18}$ : concorrono alle spese tutte quelle a lui riferibili, anche fatte dal partito (pro quota, se fatte per una pluralità di candidati: art. 7.2);

- un limite di spesa è previsto anche per ciascun partito, movimento, lista o gruppo di candidati partecipante alle elezioni (art. 10): non concorrono a raggiungerlo le spese «direttamente riferibili» a ciascun singolo candidato (art. 7.2), se fatte dal partito; tale limite si calcola moltiplicando per 200 il numero degli abitanti delle circoscrizioni (Camera) e dei collegi (Senato) in cui il partito è presente con propri candidati; si tratta dunque di un tetto non uguale per tutte le forze politiche ma proporzionato all'entità della diretta partecipazione alla competizione elettorale e, a ben vedere, piuttosto elevato (più del limite per i candidati):

- le spese relative alla campagna elettorale di candidati e partiti sono indicate a titolo probabilmente esemplificativo (ma l'elenco parrebbe tecnicamente esaustivo) dall'art. 11 della legge: alcune di quelle indicate, peraltro, sono computate forfettariamente nella misura del $30 \%$ del totale delle altre e non necessitano di documentazione (sedi, viaggi, soggiorni, posta, telefono, eventuali oneri passivi); altre sono espressamente escluse: per esempio le spese per riunioni private (non in luogo pubblico, né aperto al pubblico); inoltre sono considerate facenti parte delle spese elettorali quelle che, in realtà, precedono il $30^{\circ}$

18 Resta naturalmente la possibilità, da taluno evocata già nel dibattito parlamentare, di candidati che ottengano una pluralità di candidature al solo scopo di poter godere di un tetto aumentato entro il quale impiegare le risorse, in realtà, ai fini di una sola delle diverse candidature: ma, a parte che non si vede come impedire ciò, le candidature nelle circoscrizioni sulla base della legge 277/1993 sono così poche da far dubitare che il problema possa uscire dal novero dei casi di scuola. 
giorno dal voto, come quelle finalizzate alla presentazione delle liste; ciò avvalora l'interpretazione secondo la quale il legislatore non intende affatto far coincidere la campagna elettorale coi soli trenta giorni prima del voto; può inoltre significare che debbono essere computate nei rendiconti e consuntivi di candidati e liste le spese di cui all'art. 11 sin dall'indizione dei comizi elettorali e non solo se sostenute nei 30 giorni prima del voto: tale ipotesi, almeno per i candidati, sembra avvalorata dal dettato dell'art. 7.3 il quale prevede che ogni entrata ai fini della campagna elettorale passi attraverso il mandatario «dal giorno successivo all'indizione delle elezioni politiche»;

- per i candidati la trasparenza del bilancio è assistita da altre disposizioni: l'obbligo di raccogliere fondi solo attraverso un mandatario, garante della veridicità delle entrate, il quale è tenuto ad operare attraverso un unico apposito conto corrente (art. 7.3 e 7.6$)^{19}$; destinatario di obblighi è anche il personale di banche e uffici postali, tenuto ad identificare le generalità di coloro che versano sul conto di cui s'è detto (art. 7.4);

- secondo il modello di altre legislazioni (e sul punto anche della difforme legislazione americana) la legge dispone un tetto alle somme che possono essere versate da un singolo soggetto a vantaggio di un candidato (venti milioni, art. 7.4), la ratio è impedire che un unico soggetto possa condizionare il candidato; in questo quadro la legge aggiorna e modifica alcune disposizioni dell'art. 4 della legge 18 novembre 1981, n. 659 che si applicano anche a coloro che hanno raccolto finanziamenti per i partiti a mente dell'art. 7 della legge 2 maggio 1974, n. 195 sul finanziamento pubblico dei partiti: la rivalutazione annuale dell'importo al di sopra del quale incombe l'obbligo di dichiarazione alla Presidenza della Camera (fissato a suo tempo in $5 \mathrm{mi}$ lioni, e portata, grazie a questa previsione, a lire 10.175 .000 come stabilito da apposito decreto del Ministro dell'interno); la facoltà per i soggetti riceventi tenuti alla dichiarazione di farla, limitatamente ai soli fondi per la campagna elettorale, «a mezzo di autocertificazione» (cioè senza la sottoscrizione congiunta del finanziatore come previsto invece dalla legge 659/1981);

- si prevede inoltre che la dichiarazione di cui alla legge 5

19 Ciò vale, a mio avviso, anche nel caso in cui un candidato intenda spendere solo risorse proprie che devono a loro volta essere depositate su quel conto dal mandatario (diversa l'interpretazione di una circolare dell'Associazione bancaria che appunto non convince). 
luglio 1982, n. 441 (quella sulla pubblicità della situazione patrimoniale dei titolari di vari incarichi pubblici) (a) sia trasmessa oltre che al Presidente della Camera di appartenenza, anche al Collegio regionale di garanzia elettorale, $(b)$ corredata dal rendiconto dei contributi ricevuti e delle spese sostenute (con analitica indicazione di ogni contributo o servizio ricevuto, se da persona giuridica; solo di valore oltre i 10 milioni, se da persona fisica), nonché dagli estratti conto bancari relativi al conto aperto per la campagna; l'obbligo è esteso anche ai candidati non eletti;

- per quel che riguarda «partiti, movimenti, liste e gruppi di candidati», l'art. 12 prevede l'obbligo di presentare, a loro volta, un «consuntivo relativo alle spese per la campagna elettorale e alle relative fonti di finanziamento», senza specificazioni in ordine a certificazioni di veridicità (non c'è un mandatario); tuttavia la legge fa espresso riferimento a una documentazione da produrre «a prova delle spese stesse» (art. 12.3); copia di tale consuntivo deve essere depositata altresì presso ciascun Ufficio elettorale circoscrizionale dove il partito era presente: l'Ufficio «ne cura la pubblicità» (art. 12.4); anche tale adempimento riguarda tutti $\mathrm{i}$ soggetti collettivi partecipanti alle elezioni (non solo quelli aventi diritto ai contributi ex art. 9).

Come si è già osservato, il legislatore ha colto l'occasione della nuova legislazione sulle campagne elettorali per apportare profonde innovazioni su una materia in precedenza disciplinata in un quadro normativo diverso: i contributi per le spese elettorali, estesi, dopo duri scontri parlamentari, non solo ai partiti, alle liste, ai gruppi di candidati ma, per il solo Senato, anche ai singoli candidati.

Il 18 aprile 1993 il corpo elettorale aveva sancito la fine del finanziamento ordinario ai partiti (artt. 3 e 9 della legge 195/ 1974) ed aveva ridotto i contributi pubblici al solo rimborso delle spese elettorali (per un importo di soli 30 miliardi). Era dunque in gioco la residua possibilità di sopravvivenza organizzativa di quasi tutte le forze politiche tradizionali (ed anche di alcune di quelle più recenti). Perciò, i fautori di una più profonda ristrutturazione del sistema partitico italiano si opponevano sia a un eccessivo aumento del contributo elettorale, sia a modalità che favorissero troppo, a spese dei singoli candidati, le organizzazioni di partito; chi difendeva queste, invece, si muoveva in direzione opposta.

Ecco i tratti fondamentali della nuova disciplina: 
- vengono istituiti due fondi, uno per le elezioni della Camera, l'altro per le elezioni del Senato, pari a lire 800 per ogni abitante della Repubblica (dunque circa 45.5 miliardi l'uno) distribuiti secondo criteri coerenti con i due diversi sistemi elettorali (art. 9.1);

- il fondo per le spese elettorali per il rinnovo del Senato viene ripartito in base alla popolazione fra le 20 regioni; partecipano al riparto del fondo regionale (800 milioni ogni milione di abitanti) sulla base dei voti conseguiti: (a) i gruppi che hanno ottenuto almeno un eletto; $(b)$ i gruppi che hanno ottenuto nessun eletto ma almeno il $5 \%$ dei voti validi; $(c)$ i candidati non collegati in gruppo che siano stati eletti; $(d)$ candidati non collegati in gruppo non eletti che abbiano riportato almeno il $15 \%$ dei voti nel collegio (art. 9.2);

- premesso che, all'atto della candidatura, ciascun candidato alla Camera nella parte uninominale collegato (ai fini del c.d. «scorporo») a più liste, è obbligato a comunicare a quale lista si collega ai fini, invece, del contributo, il fondo per le spese elettorali per il rinnovo della Camera è ripartito sulla base dei soli voti riportati dalle liste nella parte proporzionale; partecipano al riparto: (a) partiti o movimenti che hanno avuto più del $4 \%$ dei voti; (b) partiti o movimenti che hanno avuto più del $3 \%$ dei voti e almeno un eletto nei collegi uninominali (art. 9.3);

- aumento a lire 800 per abitante del contributo per le elezioni Europee e riparto dei circa $45,5 \mathrm{mld}$ del relativo fondo fra partiti e movimenti con almeno un eletto in proporzione ai voti ottenuti (art. 16.3); introduzione dell'obbligo di presentazione del consuntivo di cui all'art. 12 della legge 515/93 (art. 16.5) ${ }^{20}$.

Ulteriori misure di sostegno allo sforzo dei soggetti impegnati nelle campagne elettorali sono previste, in termini di servizi, da altre disposizioni della 515/93:

20 Questa normativa non contrasta con quanto mediamente previsto in diritto comparato: in Spagna il contributo elettorale è rapportato sia ai seggi sia ai voti, ma è limitato alle forze politiche che hanno ottenuto almeno un seggio; in Francia non c'è sbarramento ma il contributo, ripartito sulla base dei voti ottenuti al primo turno, è limitato ai partiti con candidati propri in almeno 75 circoscrizioni; nel Regno Unito non vi sono contributi elettorali pubblici; in Germania v'è un finanziamento pubblico onnicomprensivo (attività ordinaria e spese elettorali) commisurato sia ai voti riportati (sbarramento dello $0,5 \%$ ), sia alla capacità di autofinanziamento, sia ai candidati presentati (almeno nel 10\% dei collegi uninominali) (sul punto v. per la Spagna La legislazione elettorale 1994; per la Francia, Masclet 1994; per la Gran Bretagna, Gardini 1994; per la Germania, Rizzoni 1994). 
- l'invio postale a tariffa agevolata di materiale in quantità pari al numero degli elettori (art. 17);

- la riduzione dell'Iva al $4 \%$ per il materiale tipografico attinente alla campagna elettorale commissionato (art. 18);

- l'obbligo per i comuni di mettere a disposizione locali adatti a riunioni politiche, a condizioni di parità e con oneri a carico degli utilizzatori (art. 19).

\section{I meccanismi di controllo}

Tutto nuovo si presenta anche il sistema dei controlli istituito dal legislatore a presidio dell'osservanza della legge 515/93. Tale sistema nasce nel corso dell'iter alla Camera dei deputati, sorretto da due intendimenti di fondo:

- escludere la competenza della magistratura ordinaria: non a caso del resto il legislatore ha rigorosamente evitato qualsiasi sanzione penale (l'unica prevista è frutto, non a caso, di un decreto governativo $)^{21}$;

- prevedere, comunque, la competenza di organi caratterizzati da sostanziale terzietà, evitando che le sanzioni amministrative siano inflitte, per esempio, da organi, come il prefetto, dipendenti dall'esecutivo.

Ciò emerge esplicitamente dai lavori parlamentari e si riflette, con poche eccezioni, nella struttura e nel funzionamento degli organi deputati al controllo e titolari del potere sanzionatorio. Vediamo:

- viene istituito, come organo permanente della durata di 4 anni, il Collegio regionale di garanzia elettorale (Crge), presieduto dal presidente della corte d'appello e composto di altri sei membri da questi nominati (rinnovabili una sola volta), tre fra $i$ magistrati ordinari (che dunque costituiscono la maggioranza numerica all'interno dell'organo) e altrettanti fra esperti (commercialisti iscritti all'albo da dieci anni, professori universitari in materie giuridiche, amministrative, economiche); non possono far parte del Collegio coloro che ricoprono o hanno ricoperto cariche politiche nei cinque anni precedenti la nomina (art.

${ }^{21}$ Si tratta di una scelta coerente con la politica giudiziaria degli ultimi anni che mira a una progressiva depenalizzazione dei reati minori: e proprio per questo di una scelta politica significativa, che si accompagna non a caso all'abolizione dell'autorizzazione a procedere penalmente contro i membri del Parlamento. 
13.2); è prevista un'indennità per i componenti non appartenenti alla magistratura (art. 13.4);

- il Crge si avvale del personale della cancelleria della corte d'appello presso la quale opera; può inoltre avvalersi della guardia di finanza; può chiedere agli uffici pubblici, compreso il Garante per la radiodiffusione e l'editoria, le notizie utili per gli accertamenti da fare (ciò vale naturalmente per gli opportuni riscontri rispetto alla documentazione che i vari soggetti destinatari delle norme della 515/93 sono tenuti a inviargli: rendiconti dei candidati, comunicazioni di editori ed emittenti tv, consuntivi di partito) (art. 13.3);

- il Crge ha il compito di «verificare la regolarità» delle dichiarazioni e dei rendiconti dei candidati, documenti del resto «liberamente consultabili» presso il Crge; ciascun elettore ha 120 giorni dalle elezioni per presentare esposti sulla loro regolarità (art. 14.2); dichiarazioni e rendiconti si considerano approvati dopo 180 giorni se il Crge non ne contesta la regolarità (art. 14.3); se lo fa, il candidato ha 15 giorni per presentare documentazione e memorie integrative (art. 14.4);

- se il deposito della dichiarazione e del rendiconto al Crge non avviene, il Crge applica la sanzione pecuniaria amministrativa da 50 a 200 milioni (art. 15.5); se il rendiconto verificato rivela una spesa superiore al tetto, il Crge applica una sanzione che può andare da un minimo pari allo sfondamento a un massimo di tre volte lo sfondamento (art. 15.6); nel caso di candidato eletto, diffidato a depositare dichiarazione e rendiconto ex art. 7.6 della 515/93, inadempiente, scatta, oltre alla sanzione pecuniaria anche la c.d. sanzione costituzionale (tanto sbandierata quanto di improbabile applicazione) della decadenza dalla carica (art. 15.8); ineleggibilità e conseguente decadenza dalla carica «con delibera della Camera di appartenenza» «ai sensi del proprio regolamento» (delibera che potrebbe non riportare i voti necessari, almeno in teoria) (art. 15.7) si aggiungono alle sanzioni pecuniari per alcune violazioni particolarmente gravi commesse da candidati eletti: oltre al caso specifico del mancato rendiconto, porta alla decadenza lo sfondamento del limite di spesa del doppio (art. 15.9); se la dichiarazione è stata presentata irregolarmente (per esempio per la assenza di documentazione o degli estratti conto) ovvero senza indicazione nominativa dei soggetti che hanno erogato contributi, il Crge applica la sanzione pecuniaria da 10 a 100 milioni; stessa sanzione nel caso di singoli contributi che abbiano superato i 20 milioni (art. 
15.11 e art. 7.4); per gli editori e per le emittenti che non presentino le comunicazioni sugli accessi in campagna elettorale (art. 8), la sanzione va da 10 a 100 milioni;

- è istituito un organo d'appello contro le decisioni del Crge presso la Corte di cassazione: si chiama Collegio centrale di garanzia elettorale ed è composto dal primo presidente della Corte (o da un presidente di sezione da lui delegato) e da sei membri scelti come i membri del Crge; decide in via definitiva entro 90 giorni (art. 14.5), dando, eventualmente, comunicazione dell'accertamento definitivo al Presidente della Camera cui appartiene il parlamentare responsabile di una violazione (art. 15.10);

- come abbiamo già visto tutte le sanzioni relative agli artt. 1 e 2 della 515/93 sono inflitte dal Garante (art. 15.1) così come quelle relative alla violazione delle disposizioni sui sondaggi (art.6), ad eccezione del caso di turbativa elettorale (art. 15.4 ) in cui interviene la magistratura ordinaria;

- per quel che riguarda le violazioni dell'art. 3: mancata indicazione del committente responsabile, mancata verifica del committente, mancata acquisizione dell'autorizzazione del candidato o del mandatario, disciplina delle affissioni) si provvede ai sensi della legge 689/1981 con sanzione pecuniaria amministrativa da 1 a 50 milioni (art. 15.2);

- diverso invece il regime dei controlli e delle sanzioni relativi alle spese elettorali dei partiti o movimenti politici, delle liste o dei gruppi di candidati (secondo la formula consueta della 515/1993); per esercitare il controllo di legittimità delle spese sostenute, infatti, la legge istituisce un apposito Collegio presso la Corte dei conti, formato da tre magistrati della Corte estratti a sorte (art. 12.2), i quali, per tutta la durata del loro incarico (nel quale sono coadiuvati da 9 revisori e dal necessario personale ausiliario) non possono assolvere ad altre funzioni (allo scopo di potersi appunto dedicare solo ai compiti assegnati dalla legge al Collegio) (art. 12.3); il controllo si esercita sui consuntivi e sulla relativa documentazione che entro 45 giorni dall'insediamento delle nuove Camere i partiti devono depositare ai Presidenti, cui incombe l'obbligo di trasmetterli alla Corte dei conti ${ }^{22}$;

22 In proposito la legge prevedeva che il deposito dovesse avvenire entro 45 giorni dall'insediamento delle Camere: poche forze politiche ottemperavano e una leggina approvata in tutta fretta (legge 15 luglio 1994, n. 448) differiva il termine al $30^{\circ}$ giorno 
- per quanto riguarda le sanzioni la legge distingue fra partiti i quali non banno diritto al contributo ex art. 9 e partiti che vi banno diritto: per i primi, il mancato deposito del consuntivo è punito dal Collegio della Corte dei conti con la sanzione amministrativa pecuniaria da 100 a 1000 milioni (art. 15.14); per i secondi sono i Presidenti delle Camere a sospendere il versamento del contributo fino al deposito del consuntivo (art. 15.13); per tutti i partiti, la mancata indicazione delle fonti di entrata della campagna elettorale è punita sempre dal Collegio della Corte dei conti con sanzione pecuniaria da 10 a 100 milioni (art. 15.15); nel caso in cui, invece, ci si trovi di fronte al superamento del limite di spesa ex art. 10, il partito non avente diritto al contributo è punito dal Collegio presso la Corte dei conti con una sanzione pecuniaria pari a un minimo della metà dello sfondamento e a un massimo del triplo (art. 15.16); il partito avente diritto al contributo, subisce analoga decurtazione del contributo stesso, inflitta dai Presidenti delle Camere.

Il sistema di controlli qui descritto incontra proprio adesso le prime applicazioni. Propongo perciò qui solo alcune considerazioni suggerite dall'analisi della legge e dalle prime esperienze. In ordine alle dichiarazioni e ai rendiconti dei candidati, sembra di poter affermare che i rendiconti presentati appaiono accurati ed esaurienti, in genere corredati di tutta la documentazione prescritta ed utile; essi denunciano spese entro i limiti previsti dalla legge: per i candidati eletti nell'ambito della Circoscrizione Toscana la media si colloca intorno ai 45 milioni a candidato, somma credibile, anche in considerazione della struttura scarsamente competitiva che le elezioni hanno avuto, nel marzo 1994, nei collegi uninominali della regione. Nel settembre 1994 i giornali hanno riferito delle prime diffide a candidati rivolte dai Crge per invitarli a consegnare o a integrare $i$ rendiconti.

Diverso il caso dei partiti: a metà luglio 1994 solo cinque forze politiche avevano depositato il proprio consuntivo presso l'Ufficio elettorale circoscrizionale di Firenze ${ }^{23}$ : Lega Nord, Pds,

dopo la pubblicazione sulla «Gazzetta ufficiale» del reparto dei fondi di cui all'art. 9 della 515/1993. Resta imprecisato il termine entro il quale, effettuati i necessari riscontri documentali, le Camere devono provvedere alla trasmissione al Collegio costituito presso la Corte dei conti.

${ }^{23}$ Fra le ragioni della mancata ottemperanza dell'obbligo di deposito presso l'Ufficio elettorale circoscrizionale dei consuntivi da parte dei partiti, secondo alcuni, il fatto 
Rifondazione comunista, Verdi, Progressisti (quando le sole liste presenti nella parte proporzionale erano 16!); si tratta di consuntivi per lo più assai accurati e puntuali, e in due casi, opportunamente, non solo complessivi ma suddivisi per circoscrizioni $^{24}$. Per quanto riguarda le spese complessive siamo certamente nei limiti della legge e della credibilità: a titolo esemplificativo, la Lega dichiara di aver speso $3,7 \mathrm{mld}$, Rifondazione 2,1, il Pds 8,5, i Verdi 0,55 (da notare che alle spese dei tre partiti di sinistra si devono aggiungere, parzialmente, le spese sostenute dai Progressisti e rendicontate a parte). I rendiconti esaminati riprendono e utilizzano la struttura delle spese elettorali quale risulta dall'art. 11 della legge 515/93.

Casi di inottemperanza rispetto al dettato della legge emergono, invece, a carico degli editori e dei titolari di concessioni o autorizzazioni alla radiodiffusione sonora o televisiva, i quali sembrano aver trascurato l'invio al Crge della comunicazione di cui all'art. 8 della legge: al punto che il Crge ha disposto un'indagine della guardia di finanza volta a chiarire le ragioni di tale diffusa inosservanza (punita con la sanzione, certo elevata per le emittenti minori, da 10 a 100 milioni) ${ }^{25}$.

Qualche difficoltà interpretativa potrebbe derivare dal mancato coordinamento fra il nuovo regime dei contributi pubblici per le spese elettorali, il regime delle sanzioni previsto dall'art. 15 e ciò che resta della legge 195/1974 sul finanziamento pubblico dei partiti come integrata dalle disposizioni della legge $659 / 1981$, con riguardo sia alle elezioni politiche, sia alle elezioni europee. Ci si può chiedere, per esempio, come si concili la

che la legge non prevederebbe sanzioni. Non sarei così sicuro di ciò: infatti al comma 13 dell'art. 15 si fa riferimento al mancato deposito dei «consuntivi» al plurale e, in ogni caso, non c'è un riferimento al deposito presso le Camere, per cui la norma la si può interpretare nel senso che il contributo è sospeso non solo quando il consuntivo non è stato depositato ai Presidenti delle Camere, ma anche quando non ne è stata depositata copia presso gli Uffici elettorali circoscrizionali.

${ }^{24} \mathrm{La}$ legge non dice nulla al riguardo: tuttavia se il deposito presso gli Uffici circoscrizionali non ha solo il fine di permettere una maggiore e più diffusa pubblicità, bensì anche quello di favorire eventuali riscontri da parte di cittadini, allora è evidente che si debbono fornire non solo consuntivi consolidati.

${ }^{25}$ I dati delle comunicazioni effettivamente inviate confermano il limitato ricorso (a pagamento) ai media sul piano locale e regionale già evidente in campagna elettorale: quotidiani come «il Tirreno» denunciano introiti da propaganda elettorale per $125 \mathrm{mi}$ lioni circa, «la Nazione» ne denuncia circa 190, emittenti tv regionali pochi milioni (1015). Ciò a fronte di spese elettorali quantificabili in alcuni miliardi complessivi e a tetti complessivi intorno ai 35 miliardi. 
sanzione della decurtazione del contributo (in caso di riscontrata violazione dei limiti di spesa) con l'avvenuta erogazione del contributo medesimo: a meno di non attendere che il Collegio presso la Corte dei conti esaurisca i suoi lavori (ma ciò può accadere oltre un anno dopo il voto, il che appare incoerente con le procedure di erogazione previste dalle norme delle precedenti leggi sul finanziamento che, per quella parte, dovrebbero essere tuttora in vigore). Si potrebbe immaginare come qualcuno intelligentemente ha fatto (v. la ricerca Cerrina Feroni) a un'erogazione in più tranches con un forte acconto e poi il saldo solo a consuntivi verificati: ma è materia che andrebbe chiarita per legge ${ }^{26}$.

\section{Conclusioni}

In conclusione: siamo di fronte a una legge non solo complessa, ma ancora in corso di prima applicazione.

Ciò rende un giudizio su di essa particolarmente difficile. Essa potrà essere giudicata solo dopo adeguata sperimentazione e sulla base di opportune ricerche: tanto più che essa è stata fatta nel modo convulso come si fanno le leggi in Italia (per cui non c'era da aspettarsi proprio da essa un drafting particolarmente accurato) ed è entrata in vigore in una fase di particolare incertezza istituzionale e politica, messa subito alla prova in una delle più aspre ma anche nuove campagne elettorali del dopoguerra (nuova per le regole del gioco; nuova per i protagonisti; nuova per l'uso delle risorse). Non basta: non è facile, nel valutare come la 515/1993 ha funzionato, stabilire quanto è dipeso da essa e quanto, piuttosto, dal contesto istituzionale e politico. Per esempio: le limitate spese elettorali quanto dipendono dall'efficacia o, al contrario, dalle maglie troppo larghe della 515/1993? Quanto dalla struttura non competitiva della contesa elettorale

${ }^{26}$ Basti pensare a quanto previsto per le elezioni europee da un lato dall'art. 3 della $659 / 1981$ che dispone l'erogazione «in un'unica soluzione entro trenta giorni dalla proclamazione dei risultati», dall'altro dall'art. 16.5 della 515/1993 che stabilisce si applichino le disposizioni di cui all'art. 12 della legge fra le quali l'obbligo di presentare il consuntivo entro 45 giorni dall'insediamento del P.E. Per le elezioni politiche un indiretto chiarimento è arrivato dalla leggina citata alla nota 22: dalla quale consegue che, prima che qualsiasi sanzione possa essere applicata, i partiti (e i candidati) avranno avuto la loro quota dei due fondi previa garanzia di una assicurazione fideiussoria. 
in molti collegi? Quanto dagli effetti indiretti di «tangentopoli» e dalla (provvisoria?) indisponibilità di certi finanziamenti?

Tutto ciò premesso, l'impressione è quella di una legge che sta funzionando, nel senso che senza di essa è ragionevole supporre che molti fenomeni lamentati si sarebbero manifestati in forme aggravate (penso in particolare alla parità d'accesso in tv) ${ }^{27}$.

Certo: anche la 515/1993 è una legge di transizione, nel senso che, proprio come le leggi elettorali del 1993, rappresenta il massimo di possibile resistenza del tradizionale Parteienprivileg affermatosi dal dopoguerra: ma è una linea di resistenza ormai arretrata, che non può fermare le trasformazioni in corso. Essa effettivamente configura una sorta di finanziamento pubblico surrettizio della politica sotto forma di contributi alle spese elettorali (triplicati, quindi sostitutivi dell'abrogato contributo annuale): una misura del resto che non mi sento di giudicare come il peggiore dei mali (è una vera misura di parità). Ma è anche una legge che ha assicurato un grado notevole di trasparenza. Mai prima di essa un cittadino avrebbe potuto conoscere in dettaglio le spese elettorali di candidati e partiti a poche settimane dal voto.

Sarà dunque opportuno verificare con molta attenzione cosa cambiare. Nessun problema per quel che riguarda le utili correzioni di discrasie e difetti minori che nella legge ci sono, e che ho cercato di segnalare, soprattutto con riferimento al necessario raccordo con la legislazione precedente. Ma per il resto chi scrive, mano a mano che approfondiva (anche in chiave comparata) lo studio della disciplina delle campagne elettorali, si andava persuadendo della necessità che il legislatore intervenga con la più grande prudenza e moderazione: evitando non solo misure che appaiono ad personam (il che è ovvio), ma anche misure che nell'impossibilità di risolvere i problemi reali (esempio: l'assetto pluralistico del sistema radiotelevisivo) pretendano di risolverne alcune conseguenze; e rinunciando alla pretesa di

27 In effetti, il legislatore era convinto di aver introdotto una disciplina dell'accesso all'informazione radiotelevisiva soddisfacente e tale da assicurare condizioni adeguate di parità. Ne sono testimonianza sia gli atti parlamentari sia le dichiarazioni sui giornali all'indomani del voto finale. Il capogruppo del Pds sen. Chiarante, per esempio, non nascondeva la sua soddisfazione per un provvedimento che conteneva «norme positive, necessarie come... in particolare quelle, certamente non gradite a Berlusconi e ai suoi amici, sulla parità d'accesso all'informazione radiotelevisiva» (v. «l'Unità» del 3 dicembre 1993). 
mettere sotto controllo l'universo dei comportamenti in campagna elettorale, finiscano di fatto solo con l'incentivare (con dannosi effetti diseducativi) sempre più sofisticate tecniche elusive.

Ciò vale anche per la questione dell'estensione temporale dell'efficacia delle norme della 515/1993 che comporterebbe la necessità di superare i problemi applicativi segnalati (par. 10): e vale ancor di più per l'eventuale disciplina dei c.d. «nuovi media» (fax, telefono 144 e 1678, marketing telefonico tout court, corrispondenza personalizzata mediante computer e stampanti laser, uso delle banche dati, uso delle reti tipo «Internet») (Vicari 1993).

Anzi, proprio alla luce di alcune delle soluzioni della 515/ 1993, mi chiedo: è possibile ed è opportuno sacrificare sull'altare di un'astratta «parità», la libertà dei cittadini singoli o associati? è possibile e opportuno, in particolare, limitare quella libertà per perseguire la tutela della autonomia di scelta elettorale del singolo quando sembra confermato che sono i cittadini più attenti e maturi quelli meglio in grado di avvalersi della più alta quantità di informazioni (in particolare diffuse via etere) in libero confronto fra loro? Una tutela uniforme degli elettori a livelli informativi più bassi, non sarebbe segno di eccessiva sfiducia e non finirebbe con l'umiliare nelle loro effettive capacità un numero di cittadini maggiore rispetto a quello dei cittadini che pretenderebbe di proteggere? Non è forse già la legislazione attuale ai limiti (se non oltre) di quella «ragionevolezza», intesa come congruità e adeguatezza rispetto al fine, di cui parla la giurisprudenza costituzionale? Non c'è il rischio di discipline irragionevolmente restrittive?

Ecco perché, molto sommessamente, suggerirei, se mai, di verificare se non è il caso di ripensare alcuni di quei divieti e di quelle limitazioni che nella legge già ci sono e che, forse, non si giustificano ${ }^{28}$.

${ }^{28}$ Penso, in particolare, al divieto di pubblicità, con deroghe incorporate, di cui all'art. 2: quando c'è già un tetto sia ai contributi singoli sia alle spese complessive di candidati e partiti; e al divieto di divulgare i sondaggi, di cui all'art. 6. 


\section{Riferimenti bibliografici}

Barile, P. (1975), Libertà di manifestazione del pensiero, Milano, Giuffrè.

- (1991), Istituzioni di diritto pubblico, Padova, Cedam.

- (1993), Finanziamento dei partiti e delle campagne elettorali in alcuni paesi stranieri, Quaderni di Documentazione del Servizio Studi, n. 2, Roma.

Camera dei Deputati (1994), Norme per l'elezione della Camera e del Senato, Quaderni di Documentazione del Servizio Studi, n. 13, Roma.

Capotosti, C.A. (1994), Garante c'è una falla, in «Il Messaggero» del 27 gennaio.

Caretti, P. (1994), Diritto pubblico dell'informazione, Bologna, Il Mulino.

Catanzaro, R. (1993), Teledemocrazia e partecipazione: molti interrogativi e prime risposte, in «Problemi dell'informazione», n. 4, pp. 379 ss.

Cerri, A. (1985), Diritto di non ascoltare l'altrui propaganda, in «Giur. cost.», I, pp. 987 ss.

Cheli, E. (1981), Pubblicità e politica: il caso italiano, in «Dir. radio e telecom.», 2, pp. 229-250.

Cicala, M. (1994), E depenalizzato il finanziamento illecito dei partiti?, in «Il corriere giuridico», n. 3 .

Cusmano, A. (1994), L'art. 20 della legge 10 dicembre 1993, n. 515: estensione delle nuove regole alle elezioni europee, regionali provinciali e comunali, in «Reg. gov. loc.», n. 1, pp. 27-36.

D'Antonio, M. e Negri, G. (1983), Il partito politico di fronte allo stato $e$ di fronte a se stesso, Milano, Giuffrè.

Di Pace, A. (a cura di) (1991), La riforma della politica: bilancio di una legislatura, in «Quaderni del circolo Rosselli», anno XI, n. 4.

Gardini, G. (1993), Commento alle norme sulla campagna elettorale, in Elezione diretta del sindaco..., coordinato da Augusto Barbera, Rimini, Maggioli, pp. 159-198.

- (1994), La disciplina delle campagne elettorali in Gran Bretagna, in «Reg. e gov. loc.», n. 1, pp. 57-78.

Gobbo, M. (1991), Lineamenti costituzionali della propaganda politica, Padova.

- (1994), La propaganda politica nell'ordinamento costituzionale statunitense, in «Reg. e gov. loc.», n. 1, pp. 99-112.

Lanchester, F. (1988), voce «Propaganda elettorale», in Enciclopedia del diritto, vol. XXXVII, Milano, Giuffrè.

- (1990), Rappresentanza, responsabilità e tecniche di espressione del suffragio. Nuovi saggi sulle votazioni, Roma, Bulzoni.

- (1993), Il nuovo attraverso il vecchio: la legge $n$. 81/1993 sull'elezione degli enti locali, in «Nomos», n. 2. 
- (1994), Le nuove regole elettorali e la carenza dei controlli, in «Reg. e gov. loc.», n. 1, pp. 7-14.

Mazzoleni, G. (1993), La comunicazione politica alla vigilia della seconda Repubblica, in «Problemi dell'informazione», n. 4, pp. 383 ss.

Morcellini, M. (a cura di) (1994), La telepolitica: polifonia e rappresentazione, in «Problemi dell'informazione», n. 2, pp. 261-283.

Mortati, C. (1976), Istituzioni di diritto pubblico, vol. II, Padova, Cedam.

Pace, A. (1992), Problematica delle libertà costituzionali. Parte speciale, Padova, Cedam.

Parlamento europeo (1986), Le financement des partis politiques dans les états membres de la Communauté, Dossier di ricerca e documentazione, Serie politica, n. 12.

Presidenza del Consiglio dei Ministri (1994), Codice elettorale 1994, Roma.

Rizzoni, G. (1994), Campagna elettorale e nuovi soggetti politici nella Repubblica federale di Germania, in «Reg. e gov. loc.», n. 1, pp. 79-97.

Rodotà, S. (1993), La sovranità nel tempo della tecnopolitica. Democrazia elettronica e democrazia rappresentativa, in «Pol. dir.», p. 573 ss.

Rodriguez, M. (1994), La comunicazione politica, in Diamanti, I. e Mannheimer, R., Milano a Roma, Roma, Donzelli.

Romano, A.A. (1989), voce «Propaganda elettorale», in Enciclopedia giuridica Treccani, Roma, Istituto della Enciclopedia Italiana.

Sani, G. (1976), Propaganda, in Bobbio, N., Matteucci, N. e Pasquino, G. (a cura di), Dizionario di politica, Torino, Utet.

Sartori, G. (19693), Democrazia e definizioni, Bologna, il Mulino.

Senato della Repubblica, (1994), La legislazione elettorale, Quaderno di Documentazione, n. 24, Roma.

Stone, G.R., Seidman, L.M., Sunstein, C.R., Tushnet, M. V. (1991), Constitutional Law, II ed., Boston-Toronto-London, Little, Brown and Company.

Tosi, S. e Fusaro, C. (1985), La via accidentata per un sistema televisivo pluralista, in «Consiglio regionale Regione Emilia Romagna», n. 1 , pp. 6-20.

Troccoli, G. (1992), Il finanziamento delle campagne elettorali, in «Quad. cost.», pp. 317 ss.

Vicari, S. (1993), Stati Uniti, Francia, Italia. Autostrade, strade e sentie$r i$, in «Problemi dell'informazione», n. 4, pp. 393-408.

Villa, M. (1994), Le amministrative 1993 a Milano: come è cambiata la comunicazione politica, in «Problemi dell'informazione.», n. 1.

Zanaroli, F. (1994), Profili sulla regolamentazione dei sondaggi pre-elettorali, in «Reg. gov. loc.», n. 1, pp. 15-25. 